\title{
DYNAMICAL INSTABILITY OF TRAPEZIUM TYPE MULTIPLE STARS
}

\author{
L.V. Mirzoyan \\ Byurakan Astrophysical Obseroatory, Armenia, Russia
}

\begin{abstract}
The properties of the Trapezium type multiple stars are discussed. The existing data confirm the dynamical instability of the Trapeziums. They show that becau se of the speci$f$ ic space configurations of components the Trapezium type multiple stars are disintegrated completely or partly (becoming ordinary systems with smaller number of components). The disintegration time is estimated some million years for Trapeziums with negative total energies and much shorter when their total energies are positive. There are some evidences in favour of the existence of Trapeziums possessing positive total energies.
\end{abstract}

\section{INTORDUCT ION}

Morphological study of multiple stars in stellar associations has shown that there exist systems among them which are dynamically instable. This significant observational fact was u sed to introduce a new type of multiple stars.

Namely, Ambartsumian [1] has divided all multiple stars into two types according to the configuration of their components. A multiple system is the Trapezium type if it contains at least three components, the mutual space distances bet ween which are of the same order of magnitude (their maximal ratio is maller than $2.5-3.0)$. Other wi se the multiple system is of the ordinary type. This criteria is valid for all multiple stars irrespective of the number of components. 
This classification of multiple stars has a profound meaning. The motions of components in the multiple stars of these two types are quite different by nature. The motions of components in the ordinary systems are Keplerian or qua siKeplerian, that is these systems can exist for a very long time. Whereas in the Trapezium type system s they are far from Keplerian motions. For this reason the Trapezium type multiple systems are dynamically instable and have to disintegrate in a very short time.

In this paper the data on the Trapezium type multiple stars are discussed.

\section{TRAPEZIUMS AND YOUNG STARS}

The Trapezium type multiple stars (Trapeziums)are similar to the open clusters by their nature.Only the numbers of components in Trapeziums are much maller.

An application of the disintegration time formula derived for clusters [2] to the Trapeziums has confirmed that they are very young [1]. For example, the disintegration time of a Trapezium having a few components of solar mass and a radius of the order of $10,000 \mathrm{a} . \mathrm{u}$. is only some million years. It is increasing slowly with the number of components in the system.

Therefore the Trapeziums of stars can be considered as very young multiple systems. Their age is not larger than some million years. Consequently they must consist of young stars.

The observations have confirmed this principle.

The Trapezium type multiple stars were found in OB-a ssociations [1].This fact was the first observational evidence in favor of their young nature.

As an illustration of this significant result a part of the Index Catalogue statistics is presented in Table 1 .

\section{TABLE 1}

The Relative Number of Real Trapeziums Among All Multiple Stars [7]

\begin{tabular}{llll}
\hline Spectrum of & Total number of & Probable number & Relative \\
the brightest & multiple stars & of Trapeziums & number of \\
components & & among them & Trapeziums
\end{tabular}
components among them

$(8)$

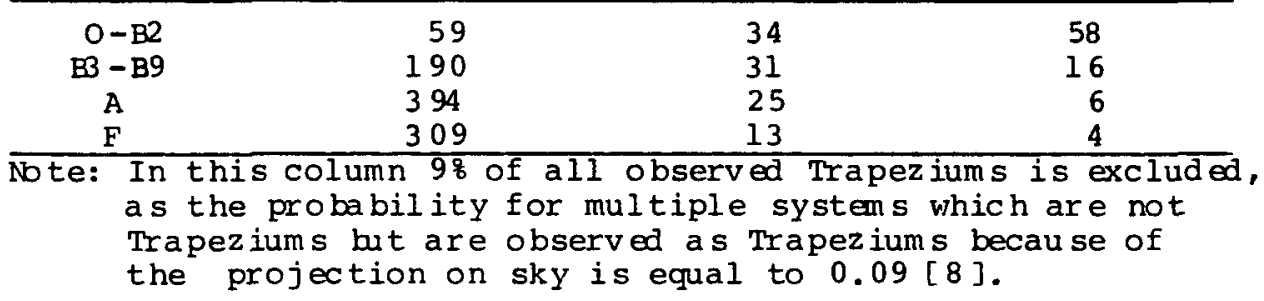


An evidence in favor of this idea was obtained from the statistics of multiple stars on the basis of Aitken's Catalogue [3]. It has been shown [4] that the majority of Trapeziums contain 0-B2 stars, as their brightest components. The statistics of multiple stars based on the Index Catalogue [5], which is more complete, has conf irmed this result [6]. This means that the real Trapeziums meet mostly among young stars.

A nother evidence in favor of the idea that Trapeziums consist of young stars was obtained by Agekian [9].Deriving the real distribution of the triple star configurations from their observed distribution he has shown that the relative number of instable triple systems (Trapeziums) among them is the largest when they contain OB-stars.

A strong tendency to conta in 0-type stars has been found by Sharpless [10] in Trapeziums connected with enission nebulae.

The high abundance of the Trapezium type multiple stars is observed also in $\mathrm{T}$-a ssociations [11] (see also Ref erences in $i t)$.

From this point of view 27 Trapeziums found by Hambarian [12] in the Orion association are of particular interest.All components of these multiple systems (triplets and quartets) consist either of the $T$ Taur $i$ and flare stars exclusively or they medley. In agreement with the disintegration of the Trapeziums the mean sizes of the systems consisting of the T Tauri stars are smaller than those of the systems consisting of flare stars only.

In connection with the disintegration of the Trapeziums it is interesting to note that Gyulbudaghian [13] has detected 19 wide multiple systems of this type which consist of the B-type stars exclusively.

It can be added that some Trapeziums are observed which consist of other very young objects: IR-sources, Herbig-Haro objects and cometary nebulae.

\section{STAR MOTIONS IN TRAPEZ IUMS}

The motions of stars in some Trapeziums containing OE-stars were studied on the basis of the position micrometric measurements for a long time.

Among them the extensive study of the Orion Trapezium carried out by Parenago [14] should be noted $f$ ir st of all. It undoubtedly testifies on its expansion during la st 120 years and therefore on the very young age of this remarka ble system. The further studies of it have confirmed these resuIts and now there is no serious reason to $c$ hange this important conclusion (see the story of studies of the orion Trapezium in [11]). 
of other studies on this problem that of silukvadze [15] is significant. Using the mea surements of the mutual angular distances between components, obta ined for about 100 years, he has studied 15 Trapeziums the brightest members of which are 0-B2 stars. Most of them can be considered as real Trapeziums. In 14 Trapeziums the expansion motions are detected on the basis of increasing distances between components $[15,16]$.

\section{DYNAMICAL EVOLUTION OF TRAPEZ IUMS}

The dynamical evolution of the Trapezium type multiple systems has been studied by Allen and Poveda [17]. The motions of stars in 30 Trapeziums having different configurations were calculated. Fach Trapezium had 6 components in tho of 15 , 20 and 50 solar masses, respectively. It was supposed that each of these systems was situated inside the sphere of 5000 a.u. radius. At last it was assumed that all of the studied Trapeziums had negative total energy.

The main result of this study is a new and completely surprising evidence in favor of the dynamical instability of Trapeziums. They have shown, that after $10^{\wedge} 6$ years of dynamical evolution only $1 / 3$ of the considered systems were disintegrated and $2 / 3$ of systems still kept their Trapezium type conf igurations.

Allen and Poveda [17] have found that this fact was in contradiction with the idea of the dynamical instability of Trapeziums, according to which the Trapezium type multiple stars possessing negative total energy must disintegrate in about $2 * 10^{\wedge} 6$ years completely or partly, losing some of their components and becoming an ordinary (stable) system [1].

Meanwhile it is easy to show that this conclusion of Allen and Poveda [17] is a result of misunder standing [18]. Indeed, according to their study of the sextet Trapeziums the probability to keep the Trapezium type configuration after $10^{\wedge} 6$ years of dynamical evolution for a Trapezium is equal to $2 / 3$. It means that after $2 * 10^{\wedge} 6$ years most of the Trapeziums considered by Allen and Poveda [17], namely 1 $(2 / 3) \quad 2=5 / 9$ part of their sample, should lo se the characteristic configurations and would no more be Trapeziums. In other words the mean age of the Trapeziums studied in [17] is of the order of $2{ }^{*} 106$ years. This is in remarkable agreement with the predicted age of such Trapeziums [1].

\section{TRAPEZIUMS AND STAR GROUP FORMATION}

As a rule all Trapeziums are observed in very young systems-stellar associations. Multiple stars situated in general galactic star field are of the ordinary type. such space distribution of multiple stars in the Galaxy is a direct consequence of the star group formation in it. 
The stars are being formed in stellar a ssociations by groups which can be both dynamically stable (ordinary type multiple stars) and dynamically instable (Trapezium type multiple stars). The Trapeziums are disintegrated before the disintegration of the maternal associations and therefore are not observed in general galactic field. On the contrary, the ordinary type multiple systems are 1 iving after the disintegration of the maternal associations and because of this reason they are observed in general galactic star field.

\section{TOTAL ENERGY OF TRAPEZIUMS}

The total energy of Trapeziums can be both negative and positive.Therefore it is impossible to judge about the size of the total energy of a system on the basis of its Trapezium configuration only. Even the Trapeziums of negative total energies had to disintegrate $[16,17]$, as the disintegration of the Trapeziums is a natural result of their space configurations irrespective of the total energy sign.

The discussion of this problem by Anbartsuma in $[1,19]$ gives every reason to a ssume that the majority of the Trapeziums have positive total enerties.

Unfortunately, the determinations of the total energy for separate Trapeziums on the basis of observational data are very incorrect. Hwever, when the differences between the calculated kinetic and potential energies are very large one can assume that in these cases the considered Trapeziums have positive total energies. For example, the systems having positive total energy probably exist among the Trapezium type multiple stars consisting of the T. Tauri type and flare stars detected by Hambarian [12] in the Orion and Hojaev [20], in the Taurus Dark Clouds.

It should be noted that the disintegration time formula is correct when the total energy of a system is negative. kowever, as we have seen, there are telling arguments to assume that some Trapeziums have positive total energies. For them the disintegration time must be much shorter [1].

\section{CONCLUSION}

The theoretical and observational data on the Trapezium type multiple stars existing at present, in particular tho se discussed in this paper, permit to suppo se that all systems of this type are disintegrated because of dynamical instability. This is why all Trapeziums are very young. The disintegration process is accelerated for Trapezium system possessing positive total energy. The age of the Trapeziums with negative energy is of the order of some million years, whereas for systems with positive total energy it is much smaller. 
It is important to note that the disintegration of such young stellar systems as Trapeziums is in line with the general tendency of cosmic evolution - the transition of matter from more dense to less dense states [21].

\section{REFERENCES}

[1] V.A. Ambartsumian, (1 954), Comm. Byurakan Obs., 15.

[2] V.A. Ambartsumian, (1 953 ), CR Acad. Sci. Armenian SSR, 16,97 .

[3] R.G. Aitken, New General Catalogue of Dou ble Stars. Carnegie Institution, Vashington, 1932 .

[4] V.A.Ambartsumian, (1951), CR ACad. SCi, Armenian SSR,13, 129 .

[5] H.M. Jeffer S, W.H. van den Boss, F.M. Greeby, (1 963 ), Index Catalogue of Visual Double Stars, Publ .: lick Obs., 21 .

[6] G.N.Salukvadze, (1 978), Bu11. Aba stumani Obs.., 49,39.

[7] L.V. Mirzoyan (1982), in 2. Kopal and J.Rahe, (eds.) Binary and Multiple Stars as Trasers of Stellar Evolut ion, Reidel, Dordrecht, p.61.

[8] V.A.Ambartsumian, (1951), CR Acad. Sci. Armenian SSR, 13,97 .

[9] T.A. Agekian (1954), Astron. Zh., 31,544.

[10] S.Sharpless, (1 954), A strophys. J.., 119,334 .

[11] G.N.Salukvadze, (1 983), A Study of the Trapezium Type Multiple Systems, Byurakan Astrophys. Obs.

[12] V.V. Hambarian (1 988 ), Astrofizika, 28,149.

[13] A.L. Gyul budaghian (1983), Astrofizika, 19,747.

[14] P.P. Par enago (1 954 ), Trudy A stron. Inst. Sternberga, 25.

[15] G.N. Salukvadze (1985), A strof izika, 22, 97.

[16] L.V. Mirzoyan, G.N. Salukvadze (1984), Astrofizika, 21, 399.

[17] C.All en, A.Poveda (1 974), in Y.Kozai (ed). The Stability of the Solar System and Small Stellar Systems, Reidel Dordrecht-Boston, p.239.

[18] L.V. Mirzoyan, M.A Mnatsakanian (1975), Astrof izika,11, 551 .

[19] V.A.Ambartsumian (1975), Observatory, 75,72.

[20] A.S. Hjaev (1985), Astrofizika, 22,425.

[21] L.V.M irzoyan (1983), in J.Ruprecht and J.Palou s, (ed s.) Star Clusters and Associations and Their Relation to the Evolution of the Galaxy, Chechoslovac Acad. Sci., Praha, p.200. 